\title{
Improved Coverings of a Square with Six and Eight Equal Circles
}

\author{
J. B. M. Melissen* and P. C. Schuuri \\ *Philips Research Laboratories, Prof. Holstlaan 4, 5656 AA Eindhoven, The Netherlands. melis- \\ sen@natlab. research.philips.com \\ †University of Twente, School of Management Studies, P.O. Box 217, 7500 AE Enschede, The Nether- \\ lands. P.C.Schuur@sms.utwente.nl
}

Submitted June 5, 1996; Accepted: October 22, 1996

\begin{abstract}
In a recent article [19], Tarnai and Gáspár used computer simulations to find thin coverings of a square with up to ten equal circles. We will give improved coverings with six and eight circles and a new, thin covering with eleven circles, found by the use of simulated annealing. Furthermore, we present a combinatorial method for constructing lower bounds for the optimal covering radius.
\end{abstract}

AMS Subject Classification: Primary 52C15

\section{Introduction}

The classical problem of packing equal circles in a square has been very popular in the literature. Since the sixties at least twenty articles have been published containing either proofs of densest packings or improvements on previous dense packings, see [2] for a partial overview.

Densest packings in a square are now known for up to 20 circles $[8,13,15,16,17,18$, $21,22]$ and for 25 and 36 circles [5, 23]. The computer-aided proof method of Peikert et al. has been extended recently up to $n=26[11,12]$. For more values than one would probably ever want to know about, extremely good packings have been found, most of which are likely to be optimal, see $[3,7,10]$.

The "dual" problem of determining thinnest coverings of a square has remained singularly devoid of attention so far. Apart from an article by Verblunsky [20], which gives a lower bound for the covering radius, there is only one recent article [19] in which Tarnai and Gáspár construct "locally optimal" circle coverings of the square with up to 10 circles. They use an engineering approach where the covering problem is transformed into the construction of an extremal bipartite graph. This graph is optimized by using a computer simulation of an equivalent shrinking bar model. No proofs of optimality are given, though. Their configurations for up to five circles and for seven circles are indeed optimal. These cases are treated in [4], as well as generalizations to a rectangle. For loosest coverings of an equilateral triangle with up to six circles, see [9]. We will show here 
that the configurations with six and eight circles as given by Tarnai and Gáspár are not optimal by presenting better coverings. Finally, we will give a new covering with eleven circles. The configurations were found by means of simulated annealing.

The paper runs as follows. Section 2 briefly discusses the methods that we used to obtain new coverings. In Section 3 the new coverings are presented. Also, in Section 4 we will demonstrate how simple combinatorial arguments can be used to obtain relevant lower bounds for the radius of the circles in a thinnest covering.

\section{The annealing approach}

To obtain an approximate solution to our covering problem via simulated annealing $[1,6]$ we place a uniform grid over the unit square. During the optimization process this grid is gradually refined. As configurations we take all the assignments of the $n$ circle centers to grid points. The cost function is chosen as the corresponding covering radius, i.e., as the smallest number $r$ such that the $n$ circles with the above centers and with radius $r$ cover the unit square. Below we shall describe how $r$ is determined.

The algorithm starts off from an arbitrary initial configuration. In each iteration a new configuration is generated by slightly perturbing the current configuration. This is done by randomly choosing one of the $n$ centers and displacing it over a small distance. The difference in cost is compared with an acceptance criterion which accepts all improvements but also admits, in a limited way, deteriorations in cost.

Initially, the acceptance criterion is taken such that deteriorations are accepted with a high probability. In this way the optimization process may be prevented from getting stuck in a local optimum. As the optimization process proceeds, the acceptance criterion is modified such that the probability for accepting deteriorations decreases. At the end of the process this probability tends to zero. The process comes to a halt when - during a prescribed number of iterations - no further improvement of the best value found so far occurs.

Let us now describe how to determine the covering radius $r$ of a given configuration. Let $U$ denote the unit square and $P=\left\{p_{i} \mid 1 \leq i \leq n\right\}$ the set of circle centers. Consider the Voronoi tessellation of $U$ [14], i.e., the "partition" of $U$ into cells obtained by assigning to each center $p$ the set $V(p)$ defined as the closure of the set of points of $U$ which are closer to $p$ than to any other center. Clearly, each cell $V(p)$ is a closed convex polyhedral set. Let $L$ denote the set consisting of all perpendicular bisectors of $p_{i} p_{j}\left(p_{i}, p_{j} \in P, i \neq j\right)$, angmented with the four lines that define the boundary of $U$. The boundary of each cell $V(p)$ is defined by lines from $L$. Let $S$ denote the set of all intersections in $U$ of any pair of lines from $L$. The covering radius is then given by

$$
r=\max _{p \in P} \max _{s \in S \cap V(p)} d(p, s)
$$


where $d$ denotes the euclidean distance. Evidently, we may rewrite this in the computationally more manageable form

$$
r=\max _{s \in S} \min _{p \in P} d(p, s)
$$

In our program the latter formula is used only once, namely for the initial configuration. From then on, $r$ is calculated incrementally, where we take advantage of the fact that only one out of $n$ centers is moved in generating a new configuration.

\section{New coverings}

The techniques described in the previous section were used to generate thin coverings. These coverings suffer from a discretization effect of the numerical method, so generally they can still be improved by analytical methods. Once the topology of the covering has been determined, it is possible to find a polynomial equation that has the optimal radius as a root. The degree of the polynomial may be very high. In this way we have been able to improve the best existing coverings with six and eight circles.

\subsection{Six circles}

An obvious, but excellent covering of the unit square with six circles is obtained by dividing the square into six equal rectangles of dimensions $\frac{1}{2}$ by $\frac{1}{3}$, and covering each small rectangle by a circle of radius $\sqrt{13} / 12=0.3004626062 \ldots$ as is shown in Fig. 1 .

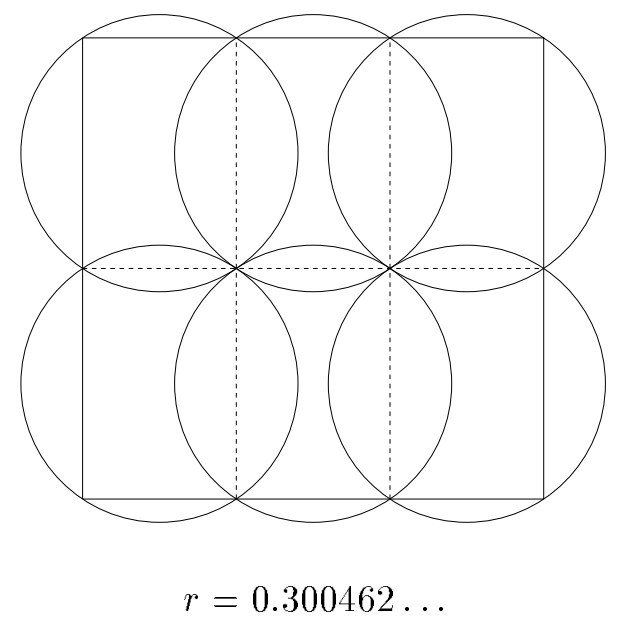

Figure 1: Obvious covering of a square with six equal circles. 
Although this covering may appear to be a reasonable candidate for the thinnest covering it is not optimal. Tarnai and Gáspár [19] recently succeeded in finding a superior covering shown in Fig. 2a with a covering radius of $0.2989506811 \ldots$

a)

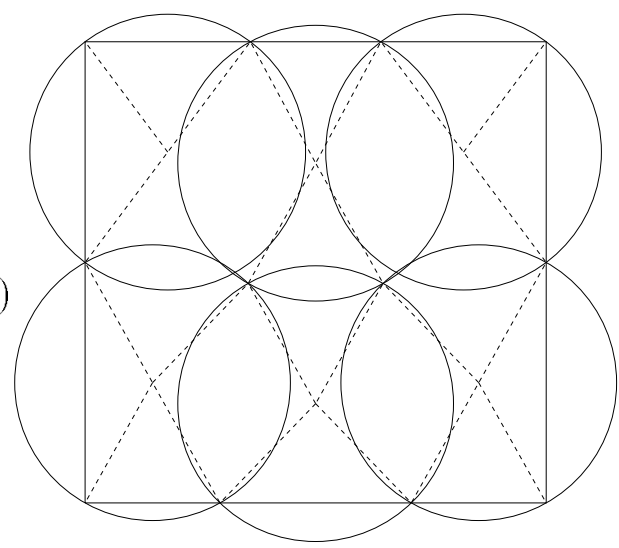

$r=0.298950 \ldots$

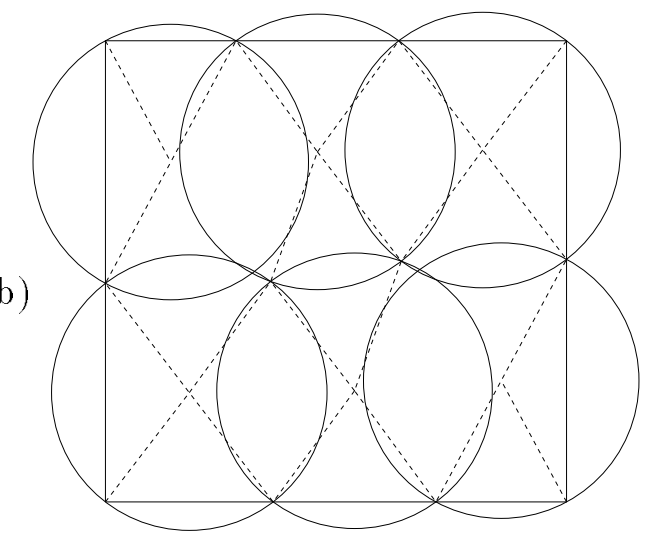

$r=0.298727 \ldots$

Figure 2: Covering with six equal circles by Tarnai and Gáspár, and the improved covering. The dotted line segments are of length $r$.

This is not the end of the story, because our annealing approach turned up an even better configuration. The covering shown in Fig. $2 \mathrm{~b}$ has a different topology than the covering found by Tarnai and Gáspár, and the covering radius is also slightly better: $0.2987270622 \ldots$ The covering of Tarnai and Gáspár has an axis of symmetry, whereas our covering is point symmetric.

\subsection{Eight circles}

The covering of the unit square with eight circles found by Tarnai and Gáspár is shown in Fig 3a. It has a covering radius of $0.2605481431 \ldots$ and has one axis of symmetry.

Using our simulated annealing approach we have found a covering which is infinitesimally better, see Fig. 3b. The configuration has two axes of symmetry. The covering radius is the smallest positive root of the following irreducible polynomial:

$$
\begin{gathered}
16-448 r+5312 r^{2}-42400 r^{3}+275368 r^{4}-1149520 r^{5}+1983264 r^{6}+1454904 r^{7}- \\
9608359 r^{8}+40979416 r^{9}-156419796 r^{10}-85939696 r^{11}+1520647364 r^{12}- \\
1614074304 r^{13}-2118065856 r^{14}+4165942016 r^{15}-20361006976 r^{16}+
\end{gathered}
$$


a)

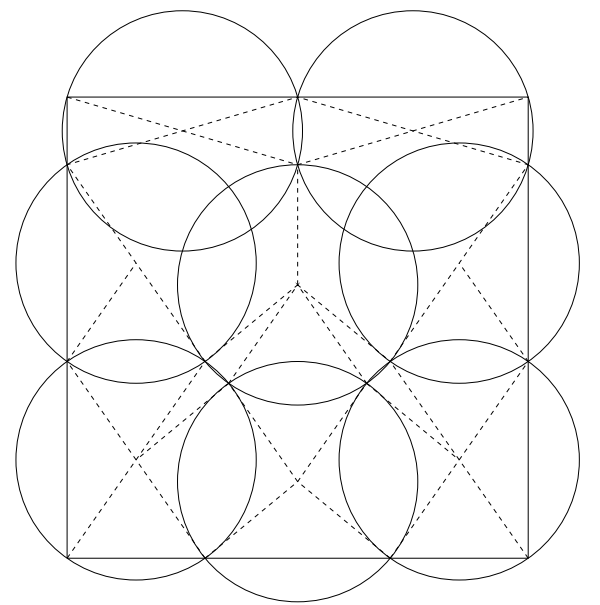

$r=0.260548 \ldots$ b)

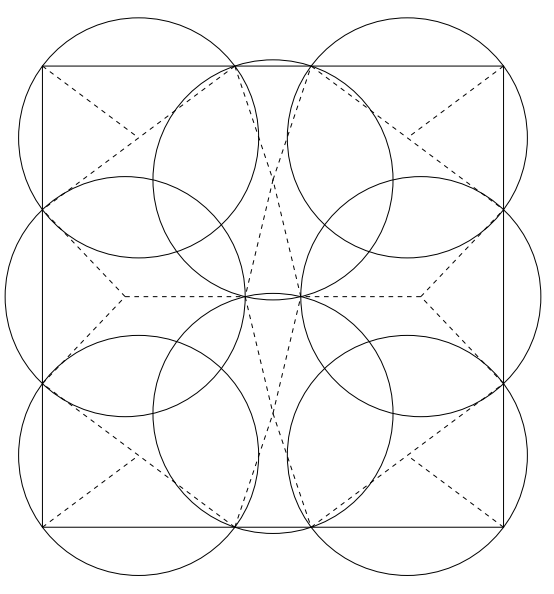

$r=0.260300 \ldots$

Figure 3: Covering of a square with eight equal circles found by Tarnai and Gáspár, and the improved covering.

$$
\begin{gathered}
48683897856 r^{17}+60092338176 r^{18}-266335027200 r^{19}+25000870912 r^{20}+ \\
495949447168 r^{21}-104762015744 r^{22}-583206961152 r^{23}-187925004288 r^{24}+ \\
974294876160 r^{25}-69986156544 r^{26}-684913065984 r^{27}+309841625088 r^{28}
\end{gathered}
$$

The numerical value of the root is $r=0.2603001058 \ldots$ The polynomial was constructed in the following way. First, we note that due to symmetry it is sufficient to determine five vertices of the pentagon that lies in a quarter of the unit square. Together with the covering radius this makes six unknowns. Unfortunately, the geometric restrictions give only five quadratic equations in these unknowns, so we have to use the fact that the covering radius must also be minimal within the above constraints. We introduce the derivatives of the unknowns with respect to one of the coordinates as new variables and differentiate the original equations. This yields eleven quadratic equations in eleven unknowns. By determining a Gröbner basis, this set can be reduced to the above equation for $r$ only.

\subsection{Eleven circles}

The best covering that we have found with eleven circles is shown in Fig. 4. The covering radius is $0.2125160164 \ldots$ The covering has two axes of symmetry. Following the best of traditions we of course conjecture this configuration to be optimal. 


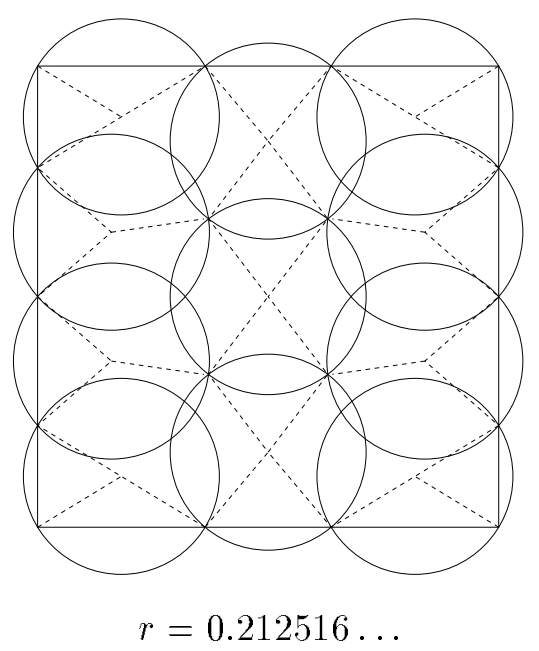

Figure 4: Covering of a square with eleven equal circles.

\section{Combinatorial methods for finding lower bounds}

Whereas upper bounds for the optimal covering radius $c_{n}$ can be obtained by "simply" providing good coverings, good lower bounds are more difficult to find. One way to construct a lower bound is as follows. Suppose that we have a covering of the unit square with $n$ circles of radius $c_{n}$. As the square can be used to tile the plane, we obtain a global circle covering of the plane with a covering density of $\pi n c_{n}^{2}$. Verblunsky [20] has shown that this density is larger than or equal to $2 \pi / \sqrt{27}$, the density of the regular hexagonal covering. This results in the following inequality.

$$
c_{n} \geq v_{n}:=\sqrt[4]{\frac{4}{27}} \frac{1}{\sqrt{n}}
$$

We will now describe a simple way, based on the pigeon hole principle, to find lower bounds for the optimal covering radius, using the results for densest circle packings. If we have $n+1$ points in a square that is covered by $n$ equal discs, then two points must be in the same disc. The smallest distance between the points is therefore a lower bound for the diameter of the discs. If the points are distributed such that the smallest distance between the points is maximal, we obtain a lower bound that is optimal for this approach. The maximum separation distance $d_{n}$ of $n$ points in the unit square is related to the densest packing radius $r_{n}$ of the unit square in the following way:

$$
d_{n}=\frac{2 r_{n}}{1-2 r_{n}} \text {. }
$$


The lower bounds resulting from the exact results that are known for the packing problem (see the references in the Introduction) are shown in the following table.

\begin{tabular}{|c|c|c|c|c|}
\hline & $\begin{array}{c}\text { upper } \\
\text { bound }\end{array}$ & \multicolumn{3}{|c|}{ lower bounds } \\
\hline$n$ & $c_{n}$ & $d_{n+1} / 2$ & $R_{2 n+1}$ & $v_{n}$ \\
\hline 1 & 0.707106 & $\mathbf{0 . 7 0 7 1 0 6}$ & $\mathbf{0 . 7 0 7 1 0 6}$ & 0.620403 \\
2 & 0.559016 & 0.517638 & $\mathbf{0 . 5 5 9 0 1 6}$ & 0.438691 \\
3 & 0.503891 & $\mathbf{0 . 5 0 0 0 0 0}$ & $\mathbf{0 . 5 0 0 0 0 0}$ & 0.358189 \\
4 & 0.353553 & $\mathbf{0 . 3 5 3 5 5 3}$ & $\mathbf{0 . 3 5 3 5 5 3}$ & 0.310201 \\
5 & 0.326160 & 0.300462 & $\mathbf{0 . 3 1 0 3 3 9}$ & 0.277452 \\
6 & 0.298727 & 0.267949 & $\mathbf{0 . 2 9 0 2 2 5}$ & 0.253278 \\
7 & 0.274291 & 0.258819 & $\mathbf{0 . 2 6 0 1 1 8}$ & 0.234490 \\
8 & 0.260300 & $\mathbf{0 . 2 5 0 0 0 0}$ & $\mathbf{0 . 2 5 0 0 0 0}$ & 0.219345 \\
9 & 0.230636 & 0.210639 & $\mathbf{0 . 2 1 6 1 7 5}$ & 0.206801 \\
10 & 0.218233 & 0.199103 & $\mathbf{0 . 2 0 4 3 6 5}$ & 0.196188 \\
11 & 0.212516 & 0.194365 & $\mathbf{0 . 1 9 5 8 4 5}$ & 0.187058 \\
\hline
\end{tabular}

A possible refinement of the previous argument would be as follows. If we take $2 n+1$ points, then one of the discs must cover at least three points. This will also give a lower bound for the covering radius. We need, however, to determine configurations of $2 n+1$ points that maximize the radius $R_{2 n+1}$ of the smallest disc that covers three of these points. The smallest circle that covers a triangle is either the circumscribed circle, or the circle that has the longest edge as diameter. If the triangle has edge lengths $a, b$ and $c$, where $a \geq b, c$, then the radius of this circle is given by

$$
\begin{aligned}
\frac{a b c}{\sqrt{s(s-a)(s-b)(s-c)}} & \text { if } a^{2}<b^{2}+c^{2}, \\
\frac{a}{2} & \text { else. }
\end{aligned}
$$

Here, $s$ is the semicircumference,

$$
s=\frac{a+b+c}{2} .
$$

Placing pairs of points on the positions of the $n+1$ points in the maximum separation configuration shows that the corresponding radius satisfies $R_{2 n+1} \geq R_{2 n+2} \geq d_{n+1} / 2$, so the lower bound obtained in this way will not be inferior to the previous bounds. An interesting question is, therefore, whether this approach can in fact improve the lower bounds found by the previous method, bearing in mind that there are no exact results available for $R_{2 n+1}$. We have found good configurations by using a multiple starting nonlinear optimization, and the results are shown in the Table. It turns out that improvement is possible in almost all cases, and that the method actually yields a proof for $n=1,2$ and 4 . 
THE EleCtronic JOURnal of COMBinatorics 3 (1996), \#R32

It should be noted that the values under $c_{n}$ in the Table are upper bounds that are only known to be sharp for $n \leq 7$, and that most of the values under $R_{2 n+1}$ are numerical values that may still be improved slightly. This, and the extension of the method to four points per circle and higher will be subject of further study. 


\section{References}

[1] E. H. L. Aarts and J. H. M. Korst, Simulated annealing and Boltzmann machines, Wiley, Chichester, 1989.

[2] H. T. Croft, K. J. Falconer, and R. K. Guy, Unsolved Problems in Geometry, Springer Verlag, Berlin, 1991, p. 111.

[3] R. L. Graham and B. D. Lubachevsky, Repeated patterns of dense packings of equal disks in a square, Electronic J. Combinatorics 3 (1996) R16. 17pp.

[4] A. Heppes and J. B. M. Melissen, Covering a rectangle with equal circles, submitted Period. Math. Hung.

[5] K. Kirchner and G. Wengerodt, Die dichteste Packung von 36 Kreisen in einem Quadrat, Beiträge Algebra Geom. 25 (1987) 147-159.

[6] S. Kirkpatrick, C. D. Gelatt Jr. and M. P. Vecchi, Optimization by simulated annealing, Science 220 (1983) 671-680.

[7] C. D. Maranas, C. A. Floudas, and P. M. Pardalos, New results in the packing of equal circles in a square, Disc. Math. 128 (1995) 187-293.

[8] J. B. M. Melissen, Densest packing of six equal circles in a square, Elem. Math. 49 (1994) 27-31.

[9] J. B. M. Melissen, Loosest circle coverings of an equilateral triangle, to appear Math. Mag. April 1997.

[10] K. J. Nurmela and P. R. J. Östergård, Packing up to 50 equal circles in a square, to appear in Discrete Comput. Geom.

[11] K. J. Nurmela and P. R. J. Östergård, Optimal packings of equal circles in a square, submitted Proceedings 8th Quadrennial International Conference on Graph Theory, Combinatorics, Algorithms and Applications (1996).

[12] K. J. Nurmela and P. R. J. Östergård, More optimal packings of equal circles in a square, in preparation.

[13] R. Peikert, Dichteste Packungen von gleichen Kreisen in einem Quadrat, Elem. Math. 49 (1994) 16-26.

[14] F. P. Preparata and M. I. Shamos, Computational Geometry, Springer Verlag, New York, 1985. 
[15] J. Schaer, The densest packing of 9 circles in a square, Canad. Math. Bull. 8 (1965) $273-277$.

[16] J. Schaer and A. Meir, On a geometric extremum problem, Canad. Math. Bull. 8 (1965) 21-27.

[17] M. Schmitz and K. Kirchner, Eine Verteilung von 13 Punkten auf einem Quadrat, Wiss. Zeitschr. Pädag. Hochschule Erfurt-Mühlhausen, 18 (1982) 113-115.

[18] B. L. Schwartz, Separating points in a square, J. Recr. Math. 3 (1970) 195-204.

[19] T. Tarnai and Z. Gáspár, Covering a square by equal circles, Elem. Math. 50 (1995), $167-170$.

[20] S. Verblunsky, On the least number of unit circles which can cover a square, $J$. London Math. Soc. 24 (1949) 164-170.

[21] G. Wengerodt, Die dichteste Packung von 16 Kreisen in einem Quadrat, Beiträge Algebra Geom. 16 (1983) 173-190.

[22] G. Wengerodt, Die dichteste Packung von 14 Kreisen in einem Quadrat, Beiträge Algebra Geom. 25 (1987) 25-46.

[23] G. Wengerodt, Die dichteste Packung von 25 Kreisen in einem Quadrat, Ann. Univ. Sci. Budapest Rolando Eötvös Sect. Math. 30 (1987) 3-15. 